\title{
FROM THE EDITORS’ DESK
}

\section{Disruptive and Deliberate Innovations in Healthcare}

\author{
Malathi Srinivasan, MD, FACP \\ Division of General Internal Medicine, Department of Medicine, University of California, Davis School of Medicine, Sacramento, CA, USA.
}

$\mathrm{J}$ Gen Intern Med 28(9):1117-8

DOI: $10.1007 / \mathrm{s} 11606-013-2550-\mathrm{x}$

(C) Society of General Internal Medicine 2013

$\mathrm{W}$ hat do we mean by innovation in health care? Clay Christensen's seminal work, "The Innovator's Prescription" expands upon his ideas of disruptive innovations that transform industries. In these new models, no organization is too big to fail, and organizations without the ability to adapt will find their missions compromised. In medicine, these discussions often leave the expert practitioner with a great sense of unease. Stepping outside of medicine may aide the uneasy expert in responding to the inexorable sweep of transformations occurring in the health care industry.

Disruptive innovations require three characteristics: technology enablers, business model innovations and value networks. Using a Christensen example, in the 1970s and 1980s, mainframe computers took deep technical engineering expertise to develop and operate, commanded high prices and were accessible to just a few privileged companies and individuals. The technology enabler, the microprocessor, allowed Steve Jobs and Michael Dell to put together desktop computers in their garages, but alone did not transform computing. The business model innovation of mass production at new processing sites allowed for lowcost, low-margin products to be developed (outside of prior large-margin sales incentive models), now affordable by the general public. Finally, the value network of mutually reinforcing innovative companies created an entirely new suite of products available to a great swathe of society. By creating products that removed the need for deep technical expertise, the technology literate can now create and edit movies at home, design their own websites, and hold teleconferences with friends and family across the world. Mainframe businesses that did not adapt or innovate were devoured by the changing technology markets which naturally adopted lower cost, similar quality, and scalable technology products.

In healthcare, new disruptive business models are exploding. Single procedure shops have dropped the cost of eye surgery or colonoscopy, shortening procedural time and increasing volumes. In hospitals, nurses perform procedures previously done only by physicians (for instance,

Published online August 1, 2013 peripherally inserted central catheter (PICC) line teams). In shopping malls and grocery chains, midlevel providers have increased access to basic and moderately complex medical care for patients. Yet medicine is not a true free market, and adoption of innovation has been tempered. Medicine's heavily regulated environment pits quality and safety concerns against process and product innovation. As Zimlichman ${ }^{1}$ points out in a recent JGIM editorial, reimbursement changes will foster creative ways of providing patient/population care, adapting to pressures surrounding bundled payments and price sensitivity from higher patient cost-sharing.

This month in JGIM, $\mathrm{Ohl}^{2}$ explores a new business model - telemedicine - that brings medical care directly to patients' hometowns by geographically disparate expert medical practitioners. For the cognitive fields, this methodology reduces expert physician overhead and increases accessibility to high quality care for internet-enabled patients and primary care providers. Ohl's small but powerful mixed-methods study demonstrates that veterans with HIV in rural Midwestern towns overwhelmingly chose telehealth care from their primary care physician offices, rather than travelling several hours to see a specialist in person for ongoing care. The telehealth technology is scalable and efficient, and a future of having routine appointments conducted from a living room, or a screening appointment from one's sickbed is a near reality.

Yet, who gets left behind? Many medically complex patients are vulnerable to poor health outcomes, especially when they cannot participate fully in their medical care. $\mathrm{Wu}^{3}$ and Bauer ${ }^{4}$ separately explore the effects of health literacy on patient outcomes. Wu's study of almost 600 CHF patients at four academic medical centers illustrates that those with low health literacy had a higher rate of rehospitalization and all-cause mortality (incidence rate ratio, 1.46). Bauer finds that amongst Kaiser patients with diabetes and depression, low health literacy rates (about $75 \%$ of their patients) had subsequently worse early/late medication persistence or had increased medication gaps. As accountable care organizations expand (accepting "full risk" for a patient's care outcomes), innovative care delivery models will likely find new ways of engaging patients and their social networks in self-care.

The lure of the disruptive innovation is compelling. Is there a counterpoint? Perhaps, less celebrated is the value of the deliberate innovation. These innovations expand the 
scope and reach of existing organizational care delivery, aligning mission, scope and finance. For example, initiatives around the patient-centered medical home (PCMH) seek to bring into alignment the needs of the patients with the processes of medical care. This transformation from "solution shops" (every problem has a unique solution) to "process shops" (most problems have a common method of approach) should increase efficiency, if supported by infrastructural and technologic innovation. Yoon ${ }^{5}$ and Cronholm $^{6}$ explore this area from a $\mathrm{PCMH}$ standpoint. In studying over 2 million patient encounters at 814 VA hospitals, Yoon found that for every $10 \%$ increase in adoption of a PCMH model, hospitalizations were reduced by $3 \%$ for ambulatory care sensitive conditions, demonstrating the value of aligning mission and care on patient outcomes. Cronholm's qualitative PCMH study illustrated the changes in mental modelsespecially around role redistribution and new role adoptionthat need to be addressed as future internal institutional transformation occurs away from clinician-centered care. Expertise may no longer be the limiting factor in high quality health care delivery.

This month, JGIM holds lessons for those who seek to create both disruptive and deliberate innovations. As synthesized information increases, and the general public becomes more informed, deep expertise may no longer be required to provide the highest quality of care. Non-experts, in conjunction with decision support, may be able to provide increasingly complex care. Technology enabled patients may take more control over their own care, while others are cared for in a more traditional manner. Fostering dissemination of cogent useful innovations, both disruptive and deliberate, is part of the Journal's charge as we look forward to the future of healthcare.

Corresponding Author: Malathi Srinivasan, MD, FACP; Division of General Internal Medicine, Department of Medicine, University of California, Davis School of Medicine, Davis, 4150 V. Street, Suite 2400, Sacramento, CA 95817, USA (e-mail: malathi@ucdavis.edu).

\section{REFERENCES}

1. Zimlichman E, Levin-Scherz J. The coming golden age of disruptive innovation in health care. JGIM. 28(7):865-7

2. Ohl M, Dilon D, Moeckli J, et al. Mixed-methods evaluation of a telehealth collaborative care program for persons with HIV infection in a rural setting. JGIM. doi:10.1007/s11606-013-2385-5.

3. Wu JR, Holmes GM, DeWalt DA, et al. Low literacy is associated with increased risk of hospitalization and death among individuals with heart failure. JGIM. doi:10.1007/s11606-013-2304-4.

4. Bauer AM, Schillinger D, Marker MM, et al. Health literacy and antidepressant medication adherence among adults with diabetes: the Diabetes Study of Northern California (DISTANCE). JGIM. doi:10.1007/ s11606-013-2402-8.

5. Yoon J, Rose DE, Canelo I, Upadhyay AS, et al. Medical home features of VHA primary care clinics and avoidable hospitalizations. JGIM. doi: 10.1007/s11606-013-2405-5.

6. Cronholm PF, Shea JA, Werner RM, et al. The patient centered medical home: mental models and culture change driving the transformation process. JGIM. doi:10.1007/s11606-013-2415-3. 